Conducting Linguistic Experiments Online with OpenSesame and OSWeb

\author{
Sebastiaan Mathôt ${ }^{1}$ and Jennifer March ${ }^{2}$ \\ ${ }^{1}$ Department of Psychology, University of Groningen, The Netherlands \\ ${ }^{2}$ Institute of Psychology, University of Hamburg, Germany
}

Address for correspondence:

Sebastiaan Mathôt

Department of Psychology

University of Groningen

Grote Kruisstraat 2/1

9712TS Groningen

The Netherlands

Email: s.mathot@,rug.nl 


\begin{abstract}
In this Methods Showcase, we outline a workflow for running behavioral experiments online, with a focus on experiments that rely on presentation of complex stimuli and measurement of reaction times, which includes many psycholinguistic experiments. The workflow that we describe here relies on three tools: OpenSesame/ OSWeb (open source) provides a user-friendly graphical interface for developing experiments; JATOS (open source) is server software for hosting experiments; and Prolific (commercial) is a platform for recruiting participants. These three tools integrate well with each other, and together provide a workflow that requires little technical expertise. We discuss, and illustrate through an example study, several challenges that are associated with running online experiments, including temporal precision, the ability to implement counterbalancing, data quality, and issues related to privacy and ethics. We conclude that these challenges are real but surmountable, and that in many cases online experiments are a viable alternative to laboratory-based experiments.
\end{abstract}

Keywords: experimental design, data collection, online experiments, methods showcase 


\section{Conducting linguistic experiments online with OpenSesame and OSWeb}

Traditionally, participants in behavioral, psycholinguistic experiments are asked to visit a psychological laboratory. There, a researcher welcomes them and provides them with a consent form. After having signed this form, participants enter a small cubicle where an experiment, typically a computer task, is waiting for them. After having completed the experiment, participants leave the cubicle and inform the researcher, who subsequently enters the cubicle to retrieve the data.

The software landscape for conducting such lab-based experiments has slowly shifted over the years. In the early 2000s, most labs were using proprietary software such as E-Prime, Inquisit, SuperLab, DirectRT, and Presentation (Stahl, 2006). With time, the software landscape has become more varied, with many labs moving towards open-source software, with PsychtoolBox (Brainard, 1997) being the first on the scene, followed later by PsychoPy (Peirce, 2007) and OpenSesame (Mathôt et al., 2012). However, despite the shift towards new tools and software, the lab-based approach remained the default for many years, and to a considerable extent it still is. In some cases, there are good reasons to conduct experiments in the lab; for example, participants may be small children or additional measures, such as eye tracking, may be collected. Moreover, experiments in which participants produce speech are still challenging (though not impossible) to implement online (e.g. Vogt et al., 2021; Wilschut et al., 2021). However, even experiments that could in principle be conducted online are often still conducted in the laboratory: of the experiments reported in Language Learning during 2020 and 2021, two experiments were conducted online, compared to eight experiments that were conducted in laboratory or laboratory-like settings yet could have been conducted online (see https://osf.io/ywbej/ for an overview). 
In a typical online experiment, participants use a web-browser to visit a web-page that contains the experiment. The main appeal of running experiments online is the potential for collecting large amounts of data in short periods of time. With online experiments, researchers no longer need to welcome participants into the laboratory one at a time; researchers need only to post a link on a participant recruitment platform, such as Prolific (prolific.co). Consider a psycholinguistic 'megastudy', such as MEGALEX (Ferrand et al., 2018), in which 197 participants spent twenty hours each (in twenty-minute sessions distributed over multiple weeks following a flexible schedule) performing a lexical-decision task in laboratory cubicles across various institutes. Could this project not have been conducted online, thus (certainly) reducing the expenses associated with hiring research assistants, travel time for participants, and maintaining laboratory space, with (hopefully) little impact on data quality? This is the promise of online experiments. And while MEGALEX is unusual in both the number of participants that were tested and the duration that each participant was tested for, the promise of increased efficiency when running experiments online also holds for smaller experiments.

When it comes to online experiments, it is important to make a distinction between questionnaire-based experiments and experiments that rely on presentation of complex stimuli and measurement of reaction times (RT). Questionnaire-based experiments, and more general experiments that can be implemented with regular web forms, have already been conducted online for some years (Munro et al., 2010). These include many linguistic experiments in which participants make judgments about words or sentences through rating scales or multiple-choice selections. Such experiments can be implemented with user-friendly online questionnaire softwares such as Qualtrics (qualtrics.com), SurveyMonkey (surveymonkey.com), or even Google Forms (google.com/forms). Here we focus on experiments that rely on presentation of 
complex stimuli and measurement of RTs, such as many psycholinguistic experiments (reviewed in Kaiser, 2013). These types of experiments have only recently started to be conducted online on a large scale. So far, three main factors have been holding back widespread adoption of online experiments of this kind.

The first limiting factor is that, until recently and in contrast to questionnaire-based experiments, developing such experiments initially required the use of advanced web technologies such as JavaScript (e.g. see an early series of online studies by Crump et al., 2013), which is the only language that web browsers are able to run natively ${ }^{1}$; yet JavaScript is not a language that many psycholinguistic researchers are familiar with. The first software package to facilitate this was jsPsych (De Leeuw, 2015), a dedicated JavaScript library for implementing psychological experiments; however, jsPsych is a software library rather than a graphical user interface, and therefore still requires knowledge of JavaScript. More recently, it has become possible to implement online experiments through graphical user interfaces, such as OpenSesame/ OSWeb (Mathôt et al., 2012; online functionality was included in 2018), PsychoPy/ PsychoJS (Peirce, 2007; online functionality was included in 2017), and Lab.JS (Henninger et al., 2021), which do not require any knowledge of JavaScript. (See Alternatives for a more comprehensive overview of software packages.) These developments have made it much easier to develop online experiments.

The second limiting factor is that online experiments require a server to distribute experiments to participants in the form of URLs, and to store the resulting participant data. Online-questionnaire software generally includes a server component out of the box; however, software packages such as OSWeb, PsychoJS, and Lab.JS do not. Initially, many researchers implemented their own ad-hoc solutions for this. However, the emergence of tools such as the open-source JATOS server 
software (Lange et al., 2015), especially in combination with the freely accessible MindProbe server ( $\underline{\text { mindprobe.eu)}}$, the free-to-use Cognition.run server (cognition.run) for jsPsych experiments, and the commercial Pavlovia server (pavlovia.org) have made also this aspect of online experimentation considerably easier.

The third limiting factor is the widespread belief among researchers that online experiments do not offer sufficient quality control, at least not for detecting small RT differences (e.g., Hamrick, in press). After all, if you don't know who your participants are, and if you don't know under which circumstances and on what kind of computers they are performing your experiments, then how can you trust your data?

The aim of this Methods Showcase is to provide a practical introduction for running behavioral experiments online, with a focus on experiments that collect RTs and accuracy. In doing so, we will touch upon all of the issues mentioned above. We will focus on a specific workflow that is centered around OpenSesame/ OSWeb and, using a semantic-categorization experiment that we recently conducted as a concrete example, we will address the specific challenges associated with psycholinguistic experiments that are of special interest to the readers of Language Learning.

\section{Workflow}

Our workflow is centered around three tools: OpenSesame/ OSWeb (Mathôt et al., 2012), JATOS (Lange et al., 2015), and Prolific. Each tool will be discussed in more detail below, but to make this section easier to understand, we start with a (p)recap of the workflow: 
1. The experiment is built with OpenSesame/ OSWeb, which is a user-friendly tool for building experiments.

2. The experiment is exported from OpenSesame/ OSWeb and imported into a JATOS server, such as MindProbe or a server that has been set up by a researcher's own organization, which is where the experiment is hosted.

3. Prolific is used to recruit participants. Prolific directs participants to the experiment on JATOS.

4. The data is downloaded from JATOS and converted to a spreadsheet format (using a conversion tool provided with OpenSesame/ OSWeb) that is convenient for data analysis.

The tools described in this section all evolve rapidly. Therefore, we will limit ourselves to those aspects of the workflow that are likely to remain valid in the near future. For implementation details, which are likely to change as tools are updated, we recommend consulting the online documentation of the tools themselves, and we provide relevant links in this article.

\section{Designing the experiment: OpenSesame/ OSWeb}

OpenSesame is a graphical experiment builder for the social sciences (Mathôt et al., 2012); it is free and open-source software that runs on Windows, Mac OS, and Linux. OpenSesame provides a comprehensive graphical user interface that allows users to implement many experiments without coding. For additional flexibility, users can also include scripting in their experiment, using either Python or JavaScript as a programming language.

OpenSesame supports presentation of complex stimuli, both visual and auditory, and collection of many kinds of response types, including key presses, screen taps, and eye movements. OpenSesame also supports advanced randomization options, including constraints, that, for 
example, allow researchers to limit the number of direct repetitions of a stimulus (or a condition), or to enforce a minimum distance between repetitions of a stimulus (or a condition). This functionality makes OpenSesame well suited for psycholinguistic studies. OpenSesame also provides limited support for questionnaires (forms); however, OpenSesame is not dedicated questionnaire software, and for questionnaire-based experiments, tools such as Qualtrics or SurveyMonkey are better suited.

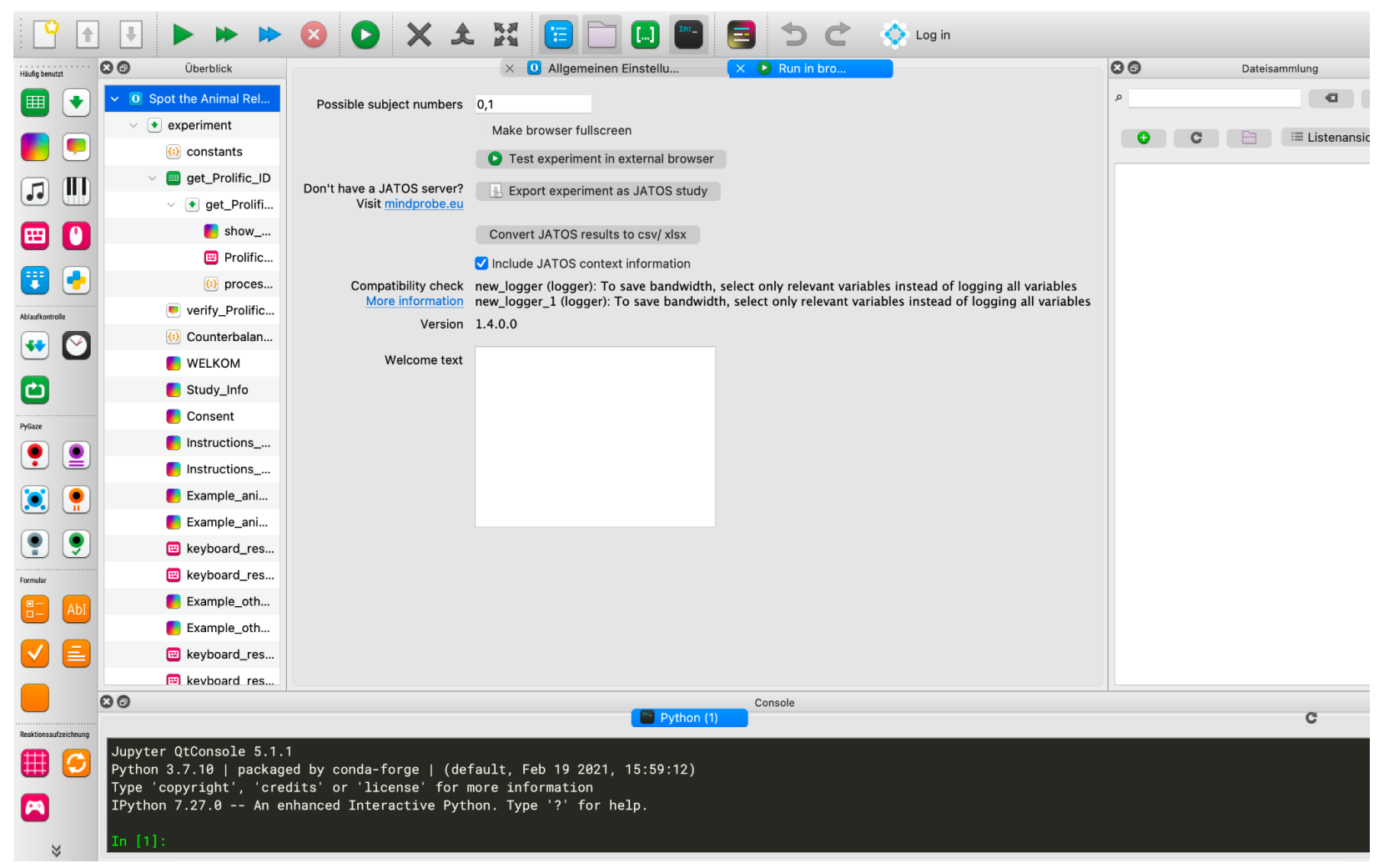

Figure 1. The OSWeb extension for OpenSesame allows you to test an experiment in a browser (without having to upload it to a server first), to export the experiment to a format that can be imported into JATOS, and to check whether the experiment is compatible with OSWeb.

OSWeb is a JavaScript application that runs OpenSesame experiments in a browser. OSWeb supports much, but not all, of the functionality that is offered by OpenSesame for lab-based 
experiments. OSWeb is pre-installed as an extension in the official OpenSesame packages (Figure 1).

An important limitation of OSWeb, as compared to OpenSesame on the desktop, is that it does not support custom Python scripts (inline_script items), which many advanced OpenSesame users rely on for developing their experiments; instead, OSWeb supports custom JavaScript (inline_javascript items). To check whether an experiment is compatible with OSWeb, users can use the "Compatibility check" that is part of the OSWeb extension. This check will highlight problems, and also suggest improvements for running experiments online, such as reducing the number of variables that are logged to save bandwidth (Figure 1).

In most cases, users can simply develop their experiment using the OpenSesame desktop application, while using the OSWeb "Compatibility check" and "Test experiment in external browser" functionality to make sure that the experiment runs in a browser. However, when the experiment is distributed to participants from a service such as Prolific, as in our example study, (or Sona Systems or Mechanical Turk) it is often useful (though not required) to log the unique identifier (e.g. the Prolific ID) that such systems assign to the participant. With this unique identifier, you can determine afterwards which result entry in JATOS (see below) corresponds to which participant on Prolific (or Sona Systems or Mechanical Turk). This is necessary if you want to be able to contact participants, withhold credit, etc. To do this, an inline_javascript item is added to the start of the experiment. This script first checks whether the experiment is hosted on a JATOS server, and if information was passed from Prolific to JATOS; if so, the script retrieves the Prolific ID from this information and logs it to the OSWeb results ${ }^{2}$. 
In summary, OpenSesame is a program for developing experiments. OSWeb is a JavaScript application for running OpenSesame experiments in a browser. Developing online experiments does not require any knowledge of web technologies; however, not all of OpenSesame's functionality is available in a browser, and users should therefore check whether their experiment is compatible with OSWeb.

Hosting the experiment: JATOS

JATOS (Lange et al., 2015) is open-source web-server software for managing online experiments. OpenSesame allows users to export their experiment to a format that can be imported into JATOS. Once an experiment has been imported, JATOS provides URLs, which are called 'workers', that can be distributed to participants (Figure 2). Participant data is also stored on JATOS. In addition to OpenSesame, JATOS also supports experiments created by a variety of other tools, such as jsPsych (De Leeuw, 2015) and lab.js (Henninger et al., 2021).

JATOS is not a single server with a single web address. Rather, it is open-source software that institutes (or individuals) can install on their own servers. The advantage of using an institute-hosted JATOS server is that this provides institutes with maximum control over where and how their experimental data is stored, thus ensuring compliance with privacy-and-ethics requirements (see also Ethics and privacy). The disadvantage is that not all researchers have access to a JATOS server, nor the technical expertise to set up their own server. To mitigate this issue, the European Society for Cognitive Psychology (https://www.escop.eu/) has sponsored the launch of MindProbe ( $\underline{\text { mindprobe.eu }}$ ), a JATOS server that is freely accessible to researchers (including non-ESCOP members). 


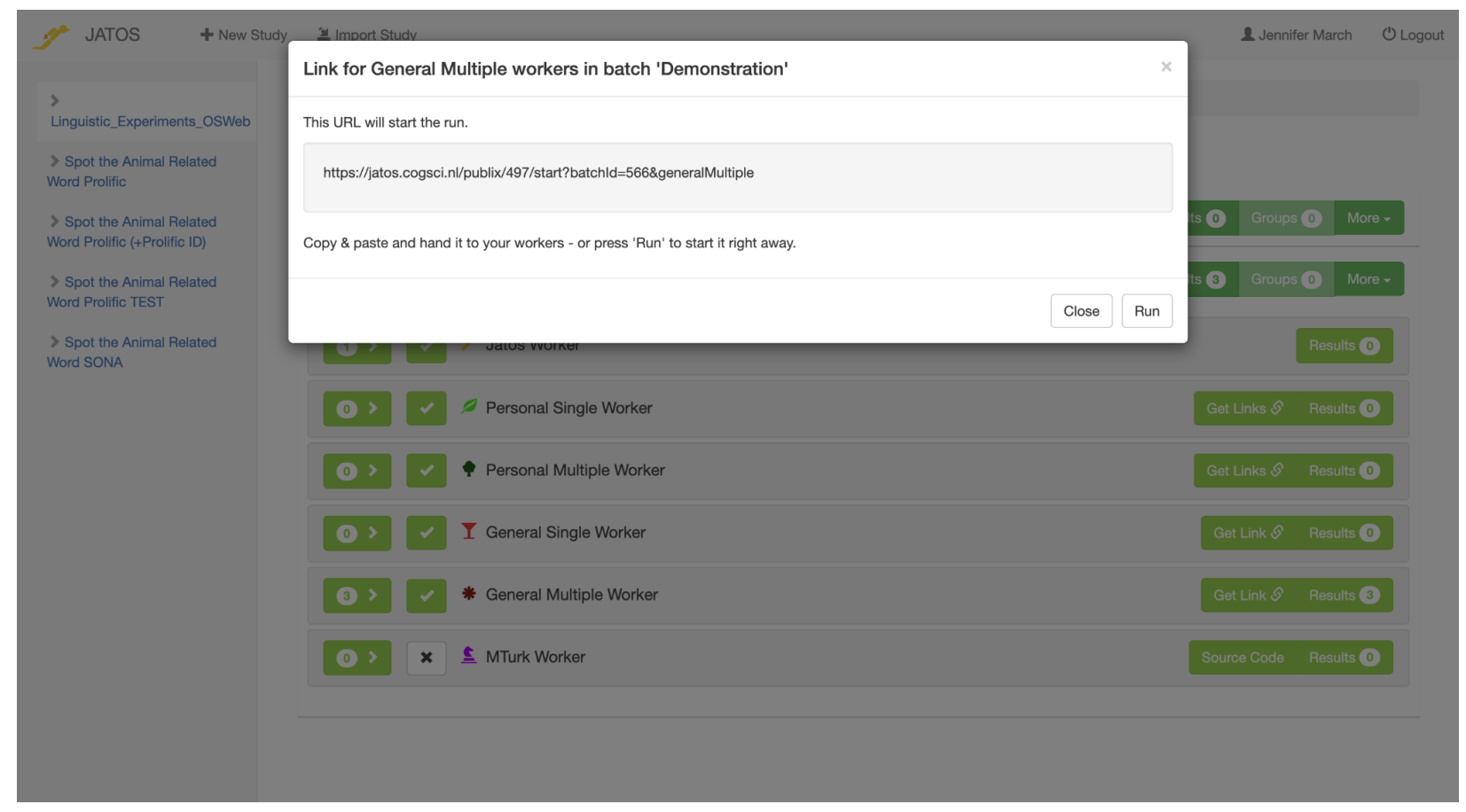

Figure 2. A JATOS server generates study URLs that can be distributed to participants. The server in this screenshot is hosted at https://jatos.cogsci.nl, but many different JATOS servers exist, typically installed by universities or research institutes on their own servers. A free JATOS server is available at https://mindprobe.eu/.

JATOS is not a platform for recruiting participants. Rather, participants are typically recruited through another platform such as, in our example experiment, Prolific (alternatives include Sona Systems and Mechanical Turk). The relationship between JATOS and a participant-recruitment platform such as Prolific can be confusing at first, but the logic is straightforward: Participants register for an online study on Prolific; from there, they are redirected to a URL that corresponds to an experiment on a JATOS server (Figure 2); when the experiment is finished, participants are redirected back to Prolific. 
In summary, JATOS is open-source web-server software for managing experiments, generating study URLs, and storing data. Institutes (or individuals) can set up their own JATOS server to retain maximum control over where and how their data is stored, or they can make use of a freely accessible server such as MindProbe.

\section{Recruiting participants: Prolific}

Prolific (https://www.prolific.co/) is a commercial platform for recruiting participants for online experiments. When researchers create a study on Prolific they indicate: how many participants they want to recruit; how long the study takes to complete; how much each participant is paid (with a suggested rate of $£ 7.50 / \$ 9.60$ per hour); and a wide variety of pre-screening criteria. For linguistic research, relevant screening criteria are related to the participant's first language, fluent languages, bilingualism, and language-related disorders. Anyone is free to sign up as a participant on Prolific after filling out a short screening form. Prolific does not provide a systematic way to inspect demographics, but the participant pool is non-representative, self-selected, and heavily skewed towards young ( $<30$ years) English-speaking participants; however, given the size of the participant pool, it is possible to find a reasonable number of participants to match most criteria, including non-English native speakers and elderly people ${ }^{3}$. For our example study (see Example experiment), we recruited only native Dutch speakers.

The communication between JATOS and Prolific involves two URLs. The first is the study URL, which is a URL on a JATOS server that should be copy-pasted into the Study Link field on Prolific (Figures 2 and 3). As mentioned above, each participant has a unique Prolific ID, which is passed to the experiment as a parameter in the study URL. The second is the end-redirect URL

(Figures 3 and 4), which is a URL on Prolific that should be copy-pasted into the Study 
Properties on JATOS. The end-redirect URL is used to direct participants back to Prolific and to let Prolific know that a study was successfully completed for a participant.

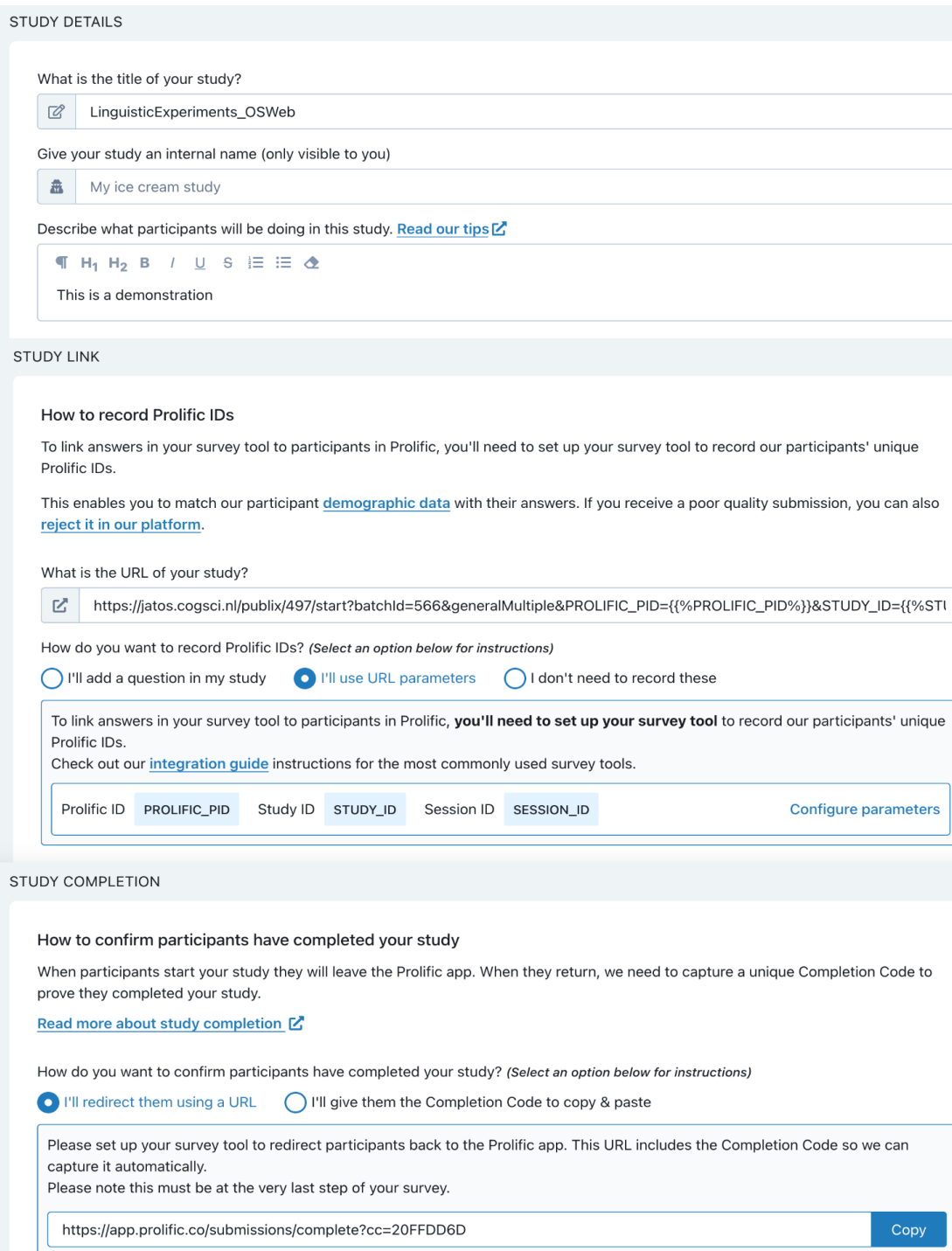

Figure 3. The study URL needs to be copied from JATOS (see Figure 2), extended with the PROLIFIC_PID, STUDY_ID, and SESSION_ID parameters, and pasted into the Study Details on Prolific. This allows Prolific to direct participants to an experiment that is hosted on JATOS. 


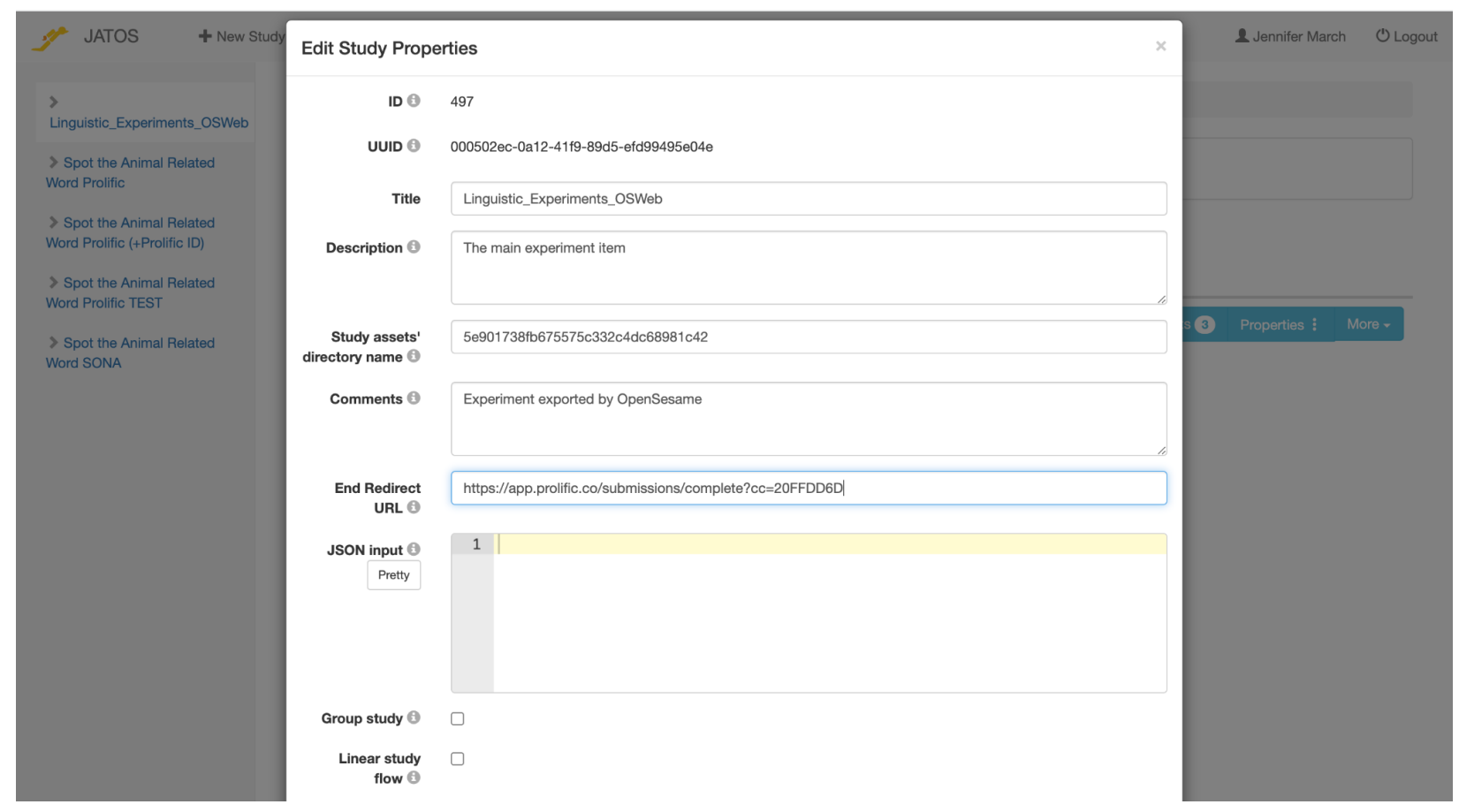

Figure 4. The end-redirect URL needs to be copied from the Study Details on Prolific, and pasted into the Study Properties on JATOS. This allows JATOS to direct participants back to Prolific when the experiment is completed. This is also how Prolific knows which participants have, and which have not, completed the experiment.

When publishing a study on Prolific, it is prudent to first test whether everything works as expected. Prolific provides a test URL that researchers can use to first test the experiment themselves from the perspective of a participant. Next, we recommend recruiting a small number of participants (e.g. five). This provides a crucial, final opportunity to check whether everything goes smoothly, before recruiting a larger sample. More specifically, this allows researchers to check whether all five participants were able to successfully complete the experiment, and if not, whether this was due to their simply abandoning the task, which happens frequently and is not necessarily a cause for concern (see Data quality), or due to a problem with the experiment (see Verifying, downloading, and processing data). 
Once a researcher is confident that the study is running smoothly, the remaining participants can be recruited. If the pre-screening criteria are not too restrictive, participant slots tend to fill up in minutes. Participants who do not complete the experiment within a specified time limit are resampled automatically by Prolific. As a result, a complete dataset is generally collected within an hour; since many participants start the experiment in parallel, the duration of data collection is roughly independent of the size of the collected dataset.

We recommend that online experiments do not take too long to complete, with a preferred duration of 15 minutes or less. This is a general recommendation for any kind of online experiment, and is based on the assumption that, with longer experiments, participants may be less likely to complete the task, and even if they do, they may lose interest and thus generate low-quality data. In addition, it is easy to collect many participants in online experiments, thus, for trial-based experiments, making it feasible to use a sampling strategy of many participants with a limited number of trials each. Brysbaert and Stevens (2018) recently estimated that a properly powered within-subject experiment requires around 1,600 observations per condition. (Clearly, this is no more than a rough rule of thumb, because statistical power depends strongly on effect size.) To reach this in a laboratory setting, researchers might favor a sampling strategy of 32 participants with 50 observations per condition each $(32 \times 50=1,600)$; in contrast, in an online experiment, researchers might favor a sampling strategy of more participants with fewer observations per participant, such as 100 participants with only 16 observations per condition each $(100 \times 16=1,600)$. Here, 'condition' refers to a single level of an experimental factor, or to a combination of levels of experimental factors in a multifactorial design.

In summary, Prolific is a commercial participant-recruitment platform for online experiments. The experiments themselves are hosted elsewhere, in the case of our example experiment on a 
JATOS server. Participants are first redirected from Prolific to the experiment, and upon completion are redirected back from the experiment to Prolific.

Verifying, downloading, and processing data

Once the desired number of participants has successfully completed the experiment, the resulting data can be viewed on, and downloaded from, JATOS. Each entry on JATOS corresponds to one experimental session, which lasts from the moment that a participant is redirected to the experiment (hosted on a JATOS server) from Prolific, until the moment that the participant is redirected back from the experiment to Prolific (Figure 5). Sessions that have been successfully completed are marked with the state FINISHED. You will often find that many entries have a different state, such as DATA_RETRIEVED or FAIL. These correspond to participants who started but did not complete the experiment, or to participants who reloaded the page (which is not allowed by JATOS), or to bugs in the experiment itself that caused the experiment to crash.

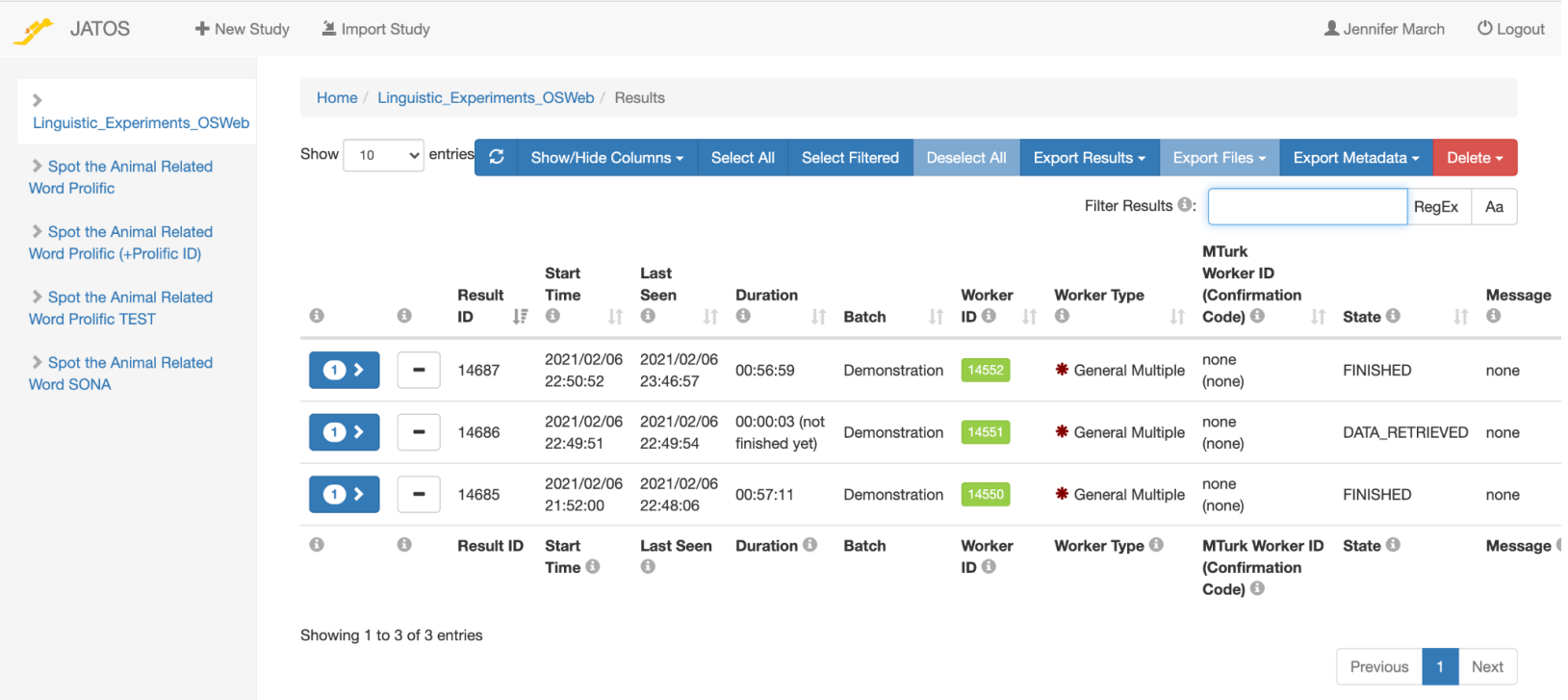


Figure 5. Each result entry on JATOS corresponds to one experimental session. The state of each result indicates whether the experiment was successfully completed (FINISHED) or not (any other state).

Because a substantial number of non-FINISHED entries is normal (see Data quality), it can be difficult to spot when something is wrong; specifically, bugs in the experiment that only affect a few participants are easily overlooked, thus resulting in affected participants unfairly missing out on their payment. To minimize the risk of this happening, and as we mentioned earlier (but it is worth repeating), it is prudent to extensively test the experiment during development (see also Designing the experiment: OpenSesame/ $O S W e b$ ) and to first recruit a small sample of participants to check whether the experiment runs smoothly, before recruiting the full sample of participants (see also Recruiting participants: Prolific).

Data from OpenSesame/ OSWeb experiments is stored in a non-standard format that cannot be read directly by commonly used software packages. To make data analysis easier, the OSWeb extension for OpenSesame provides a tool to convert JATOS results (as stored by OpenSesame/ OSWeb) to a standard . CSV or . xlsx spreadsheet that can be read by many commonly used software packages, such as R (R Core Team, 2014), JASP (JASP development team, 2021), and Microsoft Excel. For most trial-based experiments, this spreadsheet will be structured such that each row corresponds to a trial and each column corresponds to an experimental variable.

From this point onwards, researchers can analyze the data using their preferred analysis technique; an overview of all possibilities is beyond the scope of this Methods Showcase, but we will provide three common examples: first, researchers might create a pivot table in Excel and perform a (Bayesian) repeated-measures ANOVA in JASP; second, they might load the data into 
$\mathrm{R}$ and perform a linear mixed-effects (LME) analysis with lme4 (Baayen, 2008); third, they might load the data into Python and perform an LME analysis with statsmodels (Seabold \& Perktold, 2010). As we will discuss in Example experiment: Results, we have taken the third approach.

\section{Practical considerations}

\section{Data quality: Temporal precision and accuracy}

An often-voiced concern regarding online experiments is related to timing: How accurately and precisely can you control stimulus-presentation timing? And how accurately and precisely can you measure response timing? Here, "precision" refers to the variability of a measurement (its reliability), whereas "accuracy" refers to how close, on average, a measurement is to the true value (its validity).

Recent benchmark studies have measured the temporal precision of visual-stimulus presentation with different packages for online experiments (Anwyl-Irvine et al., 2020; Bridges et al., 2020; Kuroki, 2021). (There are also a number of older benchmark studies [e.g. Pinet et al., 2017; Reimers \& Stewart, 2014]. However, given the rapid developments of browser technologies, these studies are likely no longer representative.) Key metrics are the standard deviation of display durations (precision) and the mean deviation of the actual display durations from the intended display durations (accuracy). The picture that emerges from these studies is that temporal precision is decent, with some studies reporting a standard deviation of less than $5 \mathrm{~ms}$ (Bridges et al., 2020), and others reporting a slightly higher standard deviation of around $10 \mathrm{~ms}$ (Anwyl-Irvine et al., 2020). Temporal accuracy is also decent, again with some studies reporting 
lags of around $5 \mathrm{~ms}$ (Bridges et al., 2020), and others reporting lags of around $15 \mathrm{~ms}$ (Anwyl-Irvine et al., 2020). At the time of writing, the best temporal precision appears to be provided by jspysch-psychophysics (Kuroki, 2021), a plugin for jsPsych (De Leeuw, 2015), which claims standard deviations of less than $1 \mathrm{~ms}$ under some conditions, approaching the near-perfect temporal precision that can be obtained in traditional laboratory setups. In all cases, there is considerable variability between operating systems, browsers, and software packages; therefore, the values reported above are merely intended to convey a rough picture of the kind of precision and accuracy that one can realistically expect under optimal circumstances.

A key term in the preceding sentence is "optimal circumstances". The problem with timing in online experiments is not primarily that browsers are unable to offer near-perfect timing on optimal systems; rather, the problem is that most participants are not using optimal systems, which necessarily leads to less-than-perfect timing. A crucial aspect of designing a successful online experiment is therefore to choose a design that is robust to less-than-perfect timing. Fortunately, and contrary to what many researchers believe, this robustness holds for the vast majority of (behavioral) psycholinguistic studies.

Let us first consider our example experiment in which participants saw a string of one or two words, and then pressed a key on the keyboard to indicate whether this string was related to animals or not (see Example experiment: Methods). How important is temporal accuracy in this case? Not very important at all, because we are not interested in absolute RTs (remember that accuracy refers to the mean deviation of the measured value from the true value), but rather in how RTs are affected by our manipulation. And how important is temporal precision? Precision is important in the sense that decreased precision will lead to decreased statistical power (remember that precision refers to variability, or noisiness, of the measurement), that is, a 
decreased sensitivity to detect an effect of our manipulation. Therefore, less-than-perfect precision is somewhat of a problem. But one should not exaggerate this problem either, because the reduction in statistical power due to less-than-perfect precision is limited; human RTs are inherently variable (with a standard deviation of around $150 \mathrm{~ms}$ in most tasks), and therefore a small measurement error increases this natural variability only slightly (Damian, 2010; Vadillo \& Garaizar, 2016).

However, let's now consider a masked-priming experiment, such as conducted by Petit and colleagues (2006), in which a single-letter prime is presented for $33 \mathrm{~ms}$ (two frames on a typical $60 \mathrm{~Hz}$ display), preceded and followed by a mask (a '\#' character). Here, the exact presentation duration of the prime is crucial: if the prime is shown for one frame less $(16 \mathrm{~ms})$, then it may be presented too briefly to have a measurable priming effect; if the prime shown for two frames less then it is not shown at all (!); and if the prime is shown for too many frames, then it will be clearly visible, and participants will be acutely aware of the priming manipulation. Therefore, in this case, it is crucial to have both perfect temporal accuracy and precision, because the prime should be shown for exactly $33 \mathrm{~ms}$ on every trial. If not, this would not merely reduce the statistical power of the study, but invalidate its entire premise.

OpenSesame/ OSWeb automatically stores timestamps for relevant events, such as the onset of a stimulus display (sketchpad). Based on these timestamps, and assuming there is at least one stimulus in the experiment with a fixed duration, researchers can get an estimate of whether the actual presentation durations of stimuli match the intended durations. As discussed under Example Experiment: Results, in our example study we verified the presentation duration of the fixation dot to get a rough estimate of the temporal precision of our participants' systems. Importantly, these timestamps are based on 'introspection' by the browser, and are only reliable 
insofar as the browser is able to provide accurate temporal information. Therefore, these timestamps are useful for detecting extremely poor temporal precision, which in our experience is rare but does occur; however, these timestamps cannot be used to verify that temporal precision was perfect.

In summary, when running an experiment online, temporal accuracy and precision should be considered as probably-decent-but-essentially-unknown. This is not primarily because browsers are unable to offer near-perfect timing on optimal systems, but rather because most participants are not using optimal systems. For most experimental designs, imperfect timing merely reduces statistical power. However, for some experimental designs, imperfect timing poses a threat to the validity of the study. As a researcher, it is important to make sure that the experimental design is robust to imperfect timing before deciding to run it online.

\section{Counterbalancing}

"Counterbalancing", as the term is most often used, means that some factor in an experiment, such as response mapping, is systematically varied between participants to avoid it from being a confound; for example, in a lexical-decision experiment, some participants may press the right arrow key to indicate that a word was presented and the left arrow key to indicate a non-word, whereas other participants press left for words and right for non-words. Counterbalancing is distinct from factors that are varied (systematically or randomly) within a single experimental session, such as the order of trials within a block. Importantly, whereas OpenSesame/ OSWeb offers extensive support for varying factors within experimental sessions ${ }^{4}$, between-session counterbalancing of online experiments can be challenging, and we will therefore provide suggestions below. 
As a concrete example of counterbalancing (discussed in more detail below in Example experiment: Methods), in our experiment, participants saw strings that corresponded to negations (e.g. "no fish") or positive assertions (e.g. "jacket"), which was varied between blocks, rather than within blocks; that is, participants either first saw an entire block of negations followed by a block of positive assertions, or the other way around. In this counterbalancing example, half the participants started with negations, while the other half started with positive assertions, so that the effect of negation was not confounded by block order.

In an offline task, counterbalancing is often linked to the participant number, such that, for example, all even participants $(0,2, \ldots)$ start with negations and all odd participants $(1,3, \ldots)$ start with positive assertions. When running an experiment online, this approach is complicated by the fact that most systems assign a random unique identifier to each participant or each session. For example, JATOS (see Workflow) assigns a jatosResultId that is unique to each experimental session, and Prolific (see Workflow) assigns a PROLIFIC_PID that is unique to each participant but constant across sessions. Importantly, neither of these unique identifiers are linearly incrementing numbers that could be used for counterbalancing.

To work around this problem, a researcher can randomly assign a block order to each participant, and accept the risk that there may be slightly more participants starting with the negations than with the positive assertions. This, for better or worse, is the approach that we took in our example experiment, and by chance we ended up with only 20 participants who started with the negations versus 29 who started with the positive assertions - clearly an imperfectly balanced result. 
As a straightforward alternative, researchers can create two versions of the task (or more if the counterbalancing rule is more complicated) and distribute each version to an equal number of participants to achieve perfect counterbalancing.

As a more elegant and flexible alternative-but one that requires more technical skill - researchers can also make use of so-called Batch Session Data in JATOS ${ }^{5}$. Batch Session Data is a data pool that is shared between all sessions of the same "batch", where a batch generally corresponds to a single study. For our example experiment (if we had chosen to go this route), the Batch Session Data could have contained two numbers: one that indicates how many participants are still required for the negation-first block order, and one that indicates how many participants are still required for the positive-assertions-first block order. Whenever a new session is started (i.e. when a participant clicks on the experiment URL), the experiment checks the Batch Session Data to see which order requires the most participants, and then assigns this order to the current session. When both counters reach zero, the experiment stops with a message informing the participant that the experiment has already been completed. The advantage of using Batch Session Data is that it offers considerable flexibility and also allows experimental sessions to have 'memory', such that counterbalanced designs can span across multiple sessions. For example, Zhou, Lorist, and Mathôt (2021) used Batch Session Data to implement a design in which participants completed four separate experimental sessions while counterbalancing the order of these sessions between participants. 


\section{Data quality: Performance}

Another often-voiced concern regarding online experiments is related to data quality: How can researchers be sure that participants are taking an experiment seriously when they are running it by themselves on their own computers?

In our experience, the drop-out rate of participants in online experiments is far higher, in the order of 10 to $20 \%$, than in lab-based experiments, where the drop-out rate is close to $0 \%$; in other words, many participants start an online experiment but never finish it, whereas this rarely happens in the lab. In this sense, data quality is low. But since researchers can simply recruit participants until they have a sufficient (and ideally predetermined; Wagenmakers et al., 2012) number of complete datasets, a high drop-out rate is in itself not a problem. A more important question is: How reliable is the data for those participants who do finish the experiment? To assess the quality of complete and valid datasets, it is important to have some measure in the experiment that is objectively good or poor.

Let us first consider our example experiment, in which we measured RTs and response accuracy, which naturally lend themselves to assessing the quality of the data. As discussed below under Example experiment: Results, this allowed us to exclude participants for whom RTs and/ or response accuracy are outside of the 'normal range', where the normal range is often defined as two standard deviations around the grand mean RT or response accuracy. This approach is not specific to online experiments, but because data from online experiments tends to be more variable than data collected in laboratory settings, data-quality checks are even more important.

However, assessing data quality is not always as straightforward as in our example experiment. Consider an online experiment in which normative ratings are collected for a set of linguistic 
stimuli. Normative ratings do not, or least not necessarily, allow for a straightforward assessment of data quality: How can you tell whether a participant was taking the experiment seriously based on, say, how much they report the word 'sun' as having a positive valence (Mathôt et al., 2017)? In such experimental designs, it is possible to include a secondary task (an "attention check") that requires a certain amount of attention from participants in order for them to perform well, but that does not interfere with the task of interest (Kung et al., 2018). This secondary task can be as simple as occasionally presenting either a triangle or a square, and having participants quickly press a key when they see a triangle, while withholding a key press when they see a square. Participants can then be excluded when they respond too slowly (or not at all) on this task, and/ or when they have a large number of false alarms (i.e. when they press a button when no stimulus was presented). Attention checks are a useful way to verify that participants are performing a task seriously; however, they may also change how participants interpret and perform a task (Hauser \& Schwarz, 2015). Therefore, researchers should always critically assess whether attention checks are necessary in a specific experiment.

In summary, although many participants who start an online experiment do not finish it, this is not necessarily a problem as long as data quality for those participants who do finish is decent. In order to assess data quality, it is important to include some measure in the experiment, such as RT, response accuracy, or an "attention check", that indicates whether participants performed the task seriously.

\section{Privacy and ethics}

Online experiments rely on a complex digital infrastructure, and data from online experiments is often stored 'in the cloud' on physical servers of which the locations are unclear to researchers as 
well as participants. Importantly, data that is stored on third-party servers may be subject to regulations that are similarly unclear to researchers and participants. To complicate matters further, universities and research institutes often impose restrictions on where and what kind of research data can be stored. Given these complexities, how can researchers conduct online experiments while respecting privacy-and-ethics regulations?

In terms of data content, as little personally identifying information about participants as possible should be stored (Klein et al., 2018). However, even if an online experiment does not collect any personal information, the data still contains unique personal identifiers, such as the Prolific ID (see Workflow). This means that the raw data from online experiments is rarely, if ever, fully anonymous. Participants should be informed about this and actively consent to their data being used, for example by presenting a digital informed-consent form at the start of the experiment; this consent form should require a non-trivial action from participants to indicate consent, and not allow participants to start the experiment before they have done so. OpenSesame provides a form_consent item for this purpose. When publicly sharing data from online experiments, personal identifiers should be removed; for the data from our example experiment, we did this by replacing each Prolific ID by a randomly chosen number.

In terms of data storage, the optimal solution is to install JATOS on servers that are maintained by universities or research institutes themselves; this way, it is entirely clear where data is stored (at the university or research institute) and to whom it belongs (to the university or research institute). The downside of this approach is that it requires a technical department that is willing and able to set up and maintain such a server. 
If an institutional JATOS server is not available, researchers can also use third-party servers for hosting online experiments. When doing so, it is important to review the ethics-and-privacy policy of the server, and when in doubt consult a privacy officer, which are nowadays employed by most universities and researchers institutes. It is also preferable to use a server that runs open-source software, which allows for maximum transparency. MindProbe runs open-source software (JATOS); its privacy-and-ethics policy ${ }^{6}$ is intended to protect the rights of researchers and participants, and is updated occasionally based on feedback from researchers and privacy officers. However, because privacy regulations vary between countries and institutions, the responsibility to ensure that data storage complies with all regulations ultimately lies with the researcher.

\section{Example experiment}

We investigated whether word recognition is facilitated for words that are associated with brightness when they are presented in a bright font (e.g. 'sun' presented in a bright font), and for words associated with darkness when they are presented in a dark font (e.g. 'night' printed in a dark font). In other words, we asked whether word comprehension is facilitated when the semantic and sensory properties of a word are congruent; this prediction was based on embodied views of language, which posit that word meaning is in part derived from sensory and motoric representations (Glenberg \& Gallese, 2012; Pulvermüller, 2013). We further asked whether the predicted congruency effect would invert for words that are negated (e.g. interference for 'no sun' printed in bright font). Finally, we aimed to replicate the effect of negation (e.g. Dudschig \& Kaup, 2018; Kaup \& Dudschig, 2020) such that participants should respond slower to negated words ('no sun') compared to positive assertions ('sun'). 
To test this, participants performed a semantic-categorization task in which they indicated whether words were related to animals or not, a task that was unrelated to our research question, but did require semantic processing.

Similar experimental paradigms are commonly used to investigate language learning, for example by comparing different age groups (Colé et al., 1999; Grainger et al., 2012) or populations (Martens \& de Jong, 2006), or by comparing the second and first language in bilinguals (Keatley \& Gelder, 1992).

\section{Methods}

The experiment was conducted following the general workflow outlined above: first, the experiment was set up in OSWeb; second, it was hosted on JATOS; and third, Prolific was used to recruit participants. The experiment was approved by the ethics committee of the University of Groningen (PSY-1920-S-0408).

First, the experiment was implemented in OSWeb (see: Designing the experiment: OpenSesame) OSWeb). A detailed overview of the experimental design can be found in (March, 2020). In short, we investigated the effect of word type, negation and font brightness on response time. Word type included darkness-related words (e.g., "night"), brightness-related words (e.g., "sun"), animal-related words (e.g., "fish") and other/ control words (e.g., "jacket"; adapted from Mathôt et al., 2019). Half the trials included negations and the other half did not (see: Counterbalancing). We varied font brightness randomly across stimuli (i.e., stimuli were presented in either black or white font). 
On each trial, participants performed a semantic-categorization task. Specifically, participants saw a string consisting of one or two words, and indicated whether this string was associated with animals or not. The response mapping was randomly selected for each participant (i.e. randomly counterbalanced), such that some participants pressed the left arrow key for animal-associated strings and the right arrow key for other strings, while other participants pressed the right arrow key for animal-associated strings and the left arrow key for other strings.

Second, the experiment was uploaded on JATOS and connected to the recruitment plattform Prolific via a redirection URL (see: Hosting the experiment: JATOS).

Third, using Prolific's screening criteria, we recruited only Dutch native speakers and did not allow participants to sign up more than once. After participants finished the experiment hosted on JATOS, they were redirected to Prolific via a second URL (see: Recruiting participants: Prolific). Finally, data was verified, downloaded and processed as described in the corresponding section.

Results

\section{The importance of predetermined criteria}

In the section below, we show how to perform various steps to 'clean' the data from online experiments based on various criteria. These steps offer considerable degrees of freedom for researchers to steer their results in a particular direction. It is therefore good scientific practice to specify the exact steps that will be performed before data collection has taken place, for example by preregistering the analysis plan (Wagenmakers et al., 2012), and to diverge from these steps after data collection has been completed only for good reasons, which should be transparent and 
verifiable to peer reviewers. This section is intended to provide researchers with a guideline as to which steps can be taken and how.

\section{Complete and valid datasets}

Of 63 participants who signed up through Prolific, 51 participants completed the experiment. Of these 51 participants, one was excluded immediately because that participant had completed the experiment within half the time of the other participants, and an ad-hoc analysis showed that this

participant had been responding randomly. Another participant was excluded immediately because a technical issue occurred that caused part of the experiment to be repeated. This left 49 complete and valid datasets for further analysis.

\section{Data quality: Temporal precision and accuracy}

(The information that follows in this section is by necessity technical, and involves understanding the way that computer monitors are periodically refreshed. Yet this is important information for researchers who are interested in presenting visual stimuli with millisecond precision in online experiments. Readers for whom this is not a primary interest can freely skip to the next section on Data quality: performance.)

We assessed temporal precision by checking whether the actual presentation duration of the fixation dot, as logged by the browser, matched the specified presentation duration of $500 \mathrm{~ms}$. Figure 6a shows a histogram of the presentation durations of the fixation dot, measured as the timestamp of the target display minus the timestamp of the fixation-dot display as logged automatically by OSWeb. 
Within the 490 - $540 \mathrm{~ms}$ range shown in Figure 6a, the presentation duration of the fixation dot was usually somewhere in between $500 \mathrm{~ms}$ and $516 \mathrm{~ms}$, with a clear peak just short of $516 \mathrm{~ms}$. Assuming a monitor with a $60 \mathrm{~Hz}$ refresh rate, $516 \mathrm{~ms}$ corresponds to exactly 31 frames (A computer monitor is refreshed at fixed intervals, usually $16 \mathrm{~ms}$. One such refresh is called a 'frame'.) Crucially, the fact that the presentation duration was clustered around 31 frames, as opposed to being more or less uniformly distributed, suggests that on many of our participants' systems the so-called "synchronization to the vertical refresh" or "v-sync" was enabled; that is, the onset of a new visual stimulus coincided with the start of a new refresh cycle (frame) of the monitor, resulting in display durations that are multiples of the $16 \mathrm{~ms}$ frame duration. A blocking flip is generally considered optimal, because if a stimulus is presented in the middle of a refresh cycle (which happens if v-sync is not enabled), there will be a short moment during which only half the monitor shows the new stimulus, which results in a visual artifact called 'tearing' that is characterized by horizontal lines that seem to run across the monitor.

The fact that display durations are centered around $516 \mathrm{~ms}$ (31 frames), rather than $500 \mathrm{~ms}$ (30 frames), highlights the rule-of-thumb that, in OpenSesame as well as in many other software packages, you should always specify a display duration that is slightly below the intended display duration. For example, you could specify a duration of $495 \mathrm{~ms}$ for a stimulus that should be presented for $500 \mathrm{~ms}$. The rationale behind this is that, due to the discrete refresh cycles of the monitor, a presentation duration of $495 \mathrm{~ms}$ (or 29.7 frames) is impossible, and will therefore be rounded up to the next possible frame, resulting in a presentation duration of $500 \mathrm{~ms}$ (30 frames). In our example study, we did not do this, and therefore even the slightest delay resulted in a frame being skipped, thus explaining the peak around $516 \mathrm{~ms}$ ( 31 frames) as opposed to $500 \mathrm{~ms}$ (30 frames). 
Finally, the considerable variability in Figure 6a (despite a clear peak around $516 \mathrm{~ms}$ ) reflects that it is not realistic to expect single-frame temporal precision from online experiments that are conducted in uncontrolled environments.
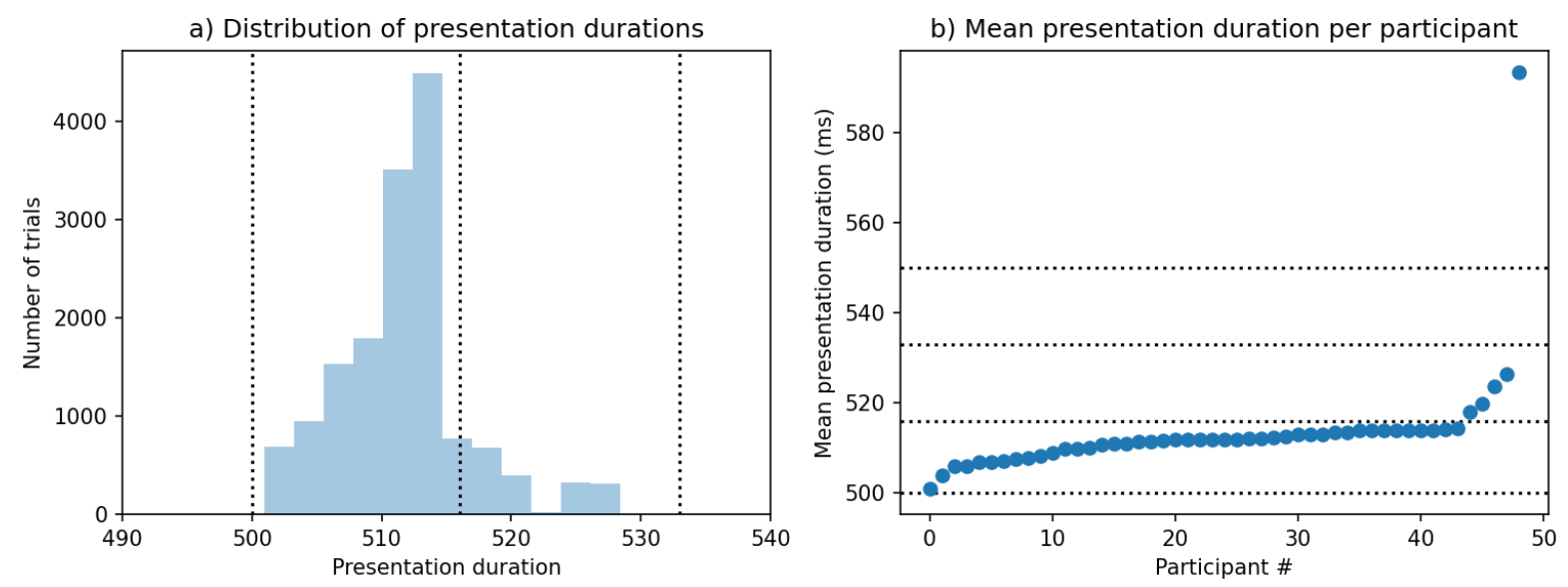

Figure 6. a) The actual duration of the fixation dot as logged by OSWeb based on browser timestamps. The mean actual duration of the fixation dot for each individual participant. Dotted lines indicate display durations that are compatible with a $60 \mathrm{~Hz}$ monitor (i.e. $500 \mathrm{~ms}$, $516 \mathrm{~ms}$, $533 \mathrm{~ms})$.

In total, only $2 \%$ of trials did not fall within the 490 - 540 ms range shown in Figure 6a. Figure. $6 \mathrm{~b}$ shows the mean presentation duration for all 49 valid participants, and clearly highlights a single participant as having exceedingly high presentation durations. Quite possibly, this participant was running the experiment on a low-performance system, or with many other programs running at the same time. This one participant accounted for all-but-one deviant presentation durations (i.e. falling outside of the range shown in Figure. 6a). Therefore we excluded this participant, leaving 48 complete, valid, and temporally precise datasets for further analysis. 


\section{Data quality: performance}

Figure 7 shows the mean RT (across both correct and incorrect trials) and accuracy for each of the 48 remaining participants. Most participants have RTs between 500 and $900 \mathrm{~ms}$, and accuracies of $70 \%$ or higher, which, based on our experience with similar experiments, is reasonable. We excluded participants with a mean RT that deviated more than two standard deviations from the grand mean RT (i.e. the mean of the per-participant mean RTs), and participants with an accuracy that deviated more than two standard deviations from the grand accuracy; based on this criterion, which is often used for studies of this kind (and, again, which should ideally be predetermined), we excluded six participants, leaving 42 participants $(13,524$ trials in total) with, at least by this standard, high-quality data.

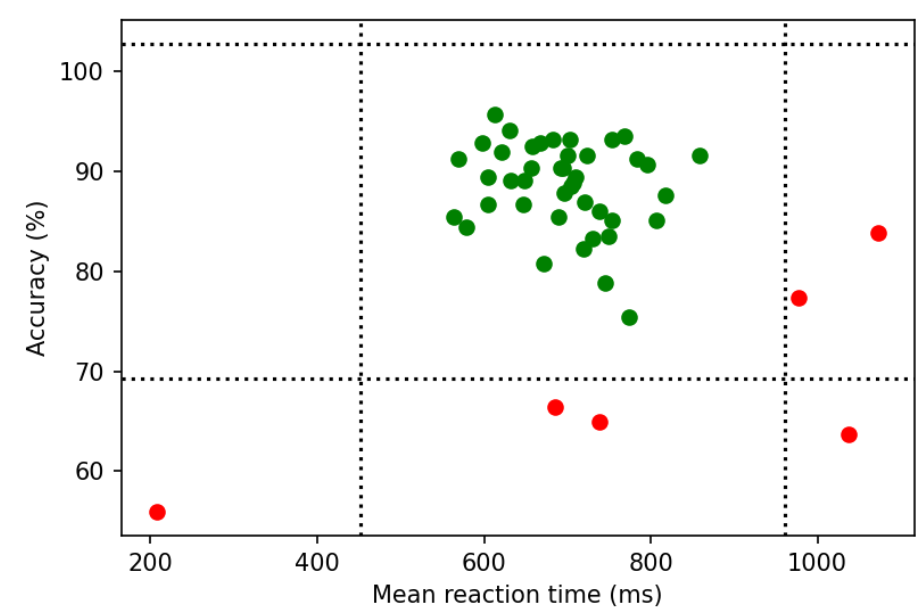

Figure 7. The mean reaction time (x axis) and response accuracy (y axis) for each participant (dots) in our example experiment. The dotted lines indicate the cut-off criteria for mean reaction time and accuracy, and participants falling outside these cut-offs (indicated in red) were excluded from further analysis. 
Next, using only words associated with brightness or darkness, we conducted an LME with response times (correct trials only) as dependent measure, semantic brightness (darkness-related or brightness-related [reference]) and font brightness (black [reference] or white) as fixed effects, and by-participant random intercepts and slopes. This revealed a (slight) effect of semantic brightness, such that brightness-related bright words were responded to faster $(z=1.97, p=.049$, $\beta=16.25,95 \% C I=[0.06,32.4])$, but no effect of font brightness $(z=0.335, p=.738, \beta=$ $-2.672,95 \% C I=[-18.31,12.97])$, nor a semantic brightness by font brightness interaction $(z=$ $0.405, p=.685, \beta=-4.66,95 \% C I=[-27.16,17.85])$. That is, we found no evidence that, in this task, word comprehension is facilitated when the semantic and sensory properties of a word are congruent.

Finally, using all words, we conducted an LME with response times (correct trials only) as dependent measure, negation (Negation vs Positive Assertion [reference]) as fixed effect, and by-participant random intercepts and slopes. This revealed an effect such that negations were responded to more slowly than positive assertions $(z=2.11, p=.035, \beta=17.11,95 \% C I=[1.24$, 32.98]), replicating this well-established effect.

\begin{abstract}
Alternatives
The workflow that we have described in this Methods Showcase is centered around OpenSesame/ OSWeb, JATOS, and Prolific. But there are many other tools that researchers can use to run experiments online, and the choice of one set of tools over another is largely one of personal preference. The different tools can be combined in various ways, resulting in a large number of possible workflows, which have recently been reviewed in Sauter and colleagues
\end{abstract}


(2020) and Grootswagers (2020). In very general terms, there are three types of tools for online experiments (commercial tools are marked with *):

- An experiment builder, where alternatives for OpenSesame/ OSWeb include jsPsych (De Leeuw, 2015), lab.js (Henninger et al., 2021), PsychoPy/ PsychoJS (Peirce, 2007), PsyToolkit (Stoet, 2010), SimplePhy (Lago, 2021), and Tatool Web (https://tatool-web.com);

- A server to host experiments, where alternatives for JATOS include Pavlovia* (https://www.pavlovia.org/), Open Lab (https://open-lab.online/), and Cognition.run (https://cognition.run);

- A platform for recruiting participants, where alternatives for Prolific* include SONA Systems* (https://www.sona-systems.com/), Amazon Mechanical Turk* (https://mturk.com/), and Qualtrics* (https://qualtrics.com/); or

- An integrated service that provides all or most of the above, where options include Gorilla* (does not provide participant recruitment; https://gorilla.sc), Inquisit Web* (https://www.millisecond.com/), Labvanced* (https://www.labvanced.com/), Resultal* (https://resultal.com/), and Testable* (https://www.testable.org/).

At the time of writing, the combination of OpenSesame/ OSWeb and JATOS is the only free and open-source workflow that allows researchers to use a graphical user interface to build and deploy online experiments that rely on complex stimuli and collection of RT and accuracy data. However, it is not the only workflow available, nor is it necessarily the optimal workflow in every situation. Below we briefly describe two main alternative workflows, both of which are currently widely used by researchers, and which are compatible with most privacy-and-ethics regulations (see the section on Ethics and privacy). 
The first alternative workflow combines PsychoPy/ PsychoJS (as a graphical user interface for building experiments) and Pavlovia (as a server for hosting experiments). This combination of tools offers similar functionality to the combination of OpenSesame/ OSWeb and JATOS; specifically, PsychoPy/ PsychoJS also offers a comprehensive graphical user interface for building complex experiments. However, Pavlovia runs proprietary software that cannot be installed on institutional servers and charges a (modest) fee for each participant tested, or for a yearly institutional license.

A second alternative workflow combines jsPsych (as a JavaScript library for building experiments) and Cognition.run (as a server for hosting experiments). This combination of tools is well suited for researchers who prefer to code their experiments directly in JavaScript using jsPsych. However, Cognition.run, while free of charge, also runs proprietary software that cannot be installed on institutional servers.

Unfortunately, at present all large-scale participant-recruitment platforms are proprietary and charge a fee. This means that all of the three workflows described above are generally combined with a commercial service such as Prolific, Mechanical Turk, or Sona Systems for the purpose of participant recruitment.

\section{Final remarks}

We have provided a practical introduction to running experiments online, with a focus on linguistic experiments that collect reaction times and response accuracy. Our workflow, which is one of many possible workflows (see e.g. Sauter et al., 2020), is centered around the use of three tools: OpenSesame/ OSWeb for developing the experiment; JATOS for hosting the experiment; and Prolific for recruiting participants. We have reviewed, and illustrated through an example 
study, several challenges associated with online experiments, related to timing, counterbalancing, data quality, and ethics and privacy. In our experience, all of these challenges are surmountable, but it is important for researchers to be aware of them before deciding to run an experiment online. In conclusion, online behavioral experiments are a viable alternative to laboratory experiments for psycholinguistic research. 


\section{Open practices statement}

Experimental scripts, analysis scripts, and anonymized data can be found at https://osf.io/ywbej/

\section{Endnotes}

${ }^{1}$ As a technical aside, there are two main alternatives to using JavaScript. First, browser extensions such as Adobe Flash and Java historically allowed web browsers to execute code written in languages other than JavaScript; however, such extensions are no longer supported by modern web browsers. Second, it is possible to 'transpile' one language, such as Python, into another language, such as JavaScript. This technique is used in some ways by both OpenSesame/ OSWeb and PsychoPy, and potentially allows online experiments to be coded in Python, a language that is more familiar than JavaScript to many researchers; however, due to fundamental differences in how languages work, transpiling is limited and error-prone. In summary, while alternatives for using JavaScript do exist, currently none of them are sufficiently well-developed and well-maintained to be a viable option for fully replacing JavaScript in online experiments.

${ }^{2}$ https://osdoc.cogsci.nl/manual/osweb/prolific

${ }^{3}$ This information is based on our trying out different screening criteria to see how many participants match.

${ }^{4}$ https://osdoc.cogsci.nl/manual/structure/loop/

${ }^{5}$ https://osdoc.cogsci.nl/manual/counterbalancing/

${ }^{6}$ https://mindprobe.eu/privacy-and-ethics.html 


\section{References}

Anwyl-Irvine, A., Dalmaijer, E. S., Hodges, N., \& Evershed, J. K. (2020). Realistic precision and accuracy of online experiment platforms, web browsers, and devices. Behavior Research Methods, 53, 1407-1425. https://doi.org/10.3758/s13428-020-01501-5

Baayen, R. H. (2008). Analyzing linguistic data: A practical introduction to statistics using $R$. Cambridge University Press.

Brainard, D. H. (1997). The psychophysics toolbox. Spatial Vision, 10(4), 433-436. https://doi.org/doi:10.1163/156856897X00357

Bridges, D., Pitiot, A., MacAskill, M. R., \& Peirce, J. W. (2020). The timing mega-study: Comparing a range of experiment generators, both lab-based and online. PeerJ, 8, e9414. https://doi.org/10.7717/peerj.9414

Brysbaert, M., \& Stevens, M. (2018). Power Analysis and Effect Size in Mixed Effects Models: A Tutorial. Journal of Cognition, 1(1), 9. https://doi.org/10.5334/joc.10

Colé, P., Annie, M., \& Grainger, J. (1999). Syllable-sized units in visual word recognition: Evidence from skilled and beginning readers of French. Applied Psycholinguistics, 20(4), 507-532. https://doi.org/10.1017/S0142716499004038

Crump, M. J. C., McDonnell, J. V., \& Gureckis, T. M. (2013). Evaluating Amazon's Mechanical Turk as a tool for experimental behavioral research. PLoS ONE, 8(3), e57410. https://doi.org/10.1371/journal.pone.0057410

Damian, M. F. (2010). Does variability in human performance outweigh imprecision in response devices such as computer keyboards? Behavior Research Methods, 42, 205-211. https://doi.org/10.3758/BRM.42.1.205

De Leeuw, J. R. (2015). jsPsych: A JavaScript library for creating behavioral experiments in a 
Web browser. Behavior Research Methods, 47(1), 1-12.

https://doi.org/10.3758/s13428-014-0458-y

Dudschig, C., \& Kaup, B. (2018). How does "not left" become "right”? Electrophysiological evidence for a dynamic conflict-bound negation processing account. Journal of Experimental Psychology: Human Perception and Performance, 44(5), 716.

Ferrand, L., Méot, A., Spinelli, E., New, B., Pallier, C., Bonin, P., Dufau, S., Mathôt, S., \& Grainger, J. (2018). MEGALEX: A megastudy of visual and auditory word recognition. Behavior Research Methods, 50(3), 1285-1307. https://doi.org/10.3758/s13428-017-0943-1

Glenberg, A. M., \& Gallese, V. (2012). Action-based language: A theory of language acquisition, comprehension, and production. Cortex, 48(7), 905-922. https://doi.org/10.1016/j.cortex.2011.04.010

Grainger, J., Lété, B., Bertand, D., Dufau, S., \& Ziegler, J. C. (2012). Evidence for multiple routes in learning to read. Cognition, 123(2), 280-292. https://doi.org/10.1016/j.cognition.2012.01.003

Grootswagers, T. (2020). A primer on running human behavioural experiments online. Behavior Research Methods, 52(2283-2286), 1-4. https://doi.org/10.3758/s13428-020-01395-3

Hamrick, P. (in press). Conducting reaction time research in L2 psycholinguistics. In A. Godfroid \& H. Hopp (Eds.), The Routledge handbook of SLA and psycholinguistics. Routledge.

Hauser, D. J., \& Schwarz, N. (2015). It's a trap! Instructional manipulation checks prompt systematic thinking on "tricky" tasks. Sage Open, 5(2). https://doi.org/10.1177/2158244015584617

Henninger, F., Shevchenko, Y., Mertens, U., Kieslich, P. J., \& Hilbig, B. E. (2021). lab. js: A 
free, open, online study builder. Behavior Research Methods.

https://doi.org/10.3758/s13428-019-01283-5

JASP development team. (2021). JASP (0.16) [Computer software]. https://jasp-stats.org/

Kaiser, E. (2013). Experimental paradigms in psycholinguistics. In R. J. Podesva \& D. Sharma (Eds.), Research methods in linguistics (pp. 135-168). Cambridge University Press.

Kaup, B., \& Dudschig, C. (2020). Understanding negation. In The Oxford handbook of negation.

Keatley, C., \& Gelder, B. de. (1992). The bilingual primed lexical decision task: Cross-language priming disappears with speeded responses. European Journal of Cognitive Psychology, 4(4), 273-292. https://doi.org/10.1080/09541449208406188

Klein, O., Hardwicke, T. E., Aust, F., Breuer, J., Danielsson, H., Mohr, A. H., IJzerman, H., Nilsonne, G., Vanpaemel, W., \& Frank, M. C. (2018). A practical guide for transparency in psychological science. Collabra: Psychology, 4(1), 20. https://doi.org/10.1525/collabra.158

Kung, F. Y. H., Kwok, N., \& Brown, D. J. (2018). Are attention check questions a threat to scale validity? Applied Psychology, 67(2), 264-283. https://doi.org/10.1111/apps.12108

Kuroki, D. (2021). A new jsPsych plugin for psychophysics, providing accurate display duration and stimulus onset asynchrony. Behavior Research Methods, 53, 301-310. https://doi.org/10.3758/s13428-020-01445-w

Lago, M. A. (2021). SimplePhy: An open-source tool for quick online perception experiments. Behavior Research Methods, 53, 1669-1676. https://doi.org/10.3758/s13428-020-01515-z

Lange, K., Kühn, S., \& Filevich, E. (2015). "Just Another Tool for Online Studies"(JATOS): An easy solution for setup and management of web servers supporting online studies. PloS 
One, 10(6), e0130834. https://doi.org/10.1371/journal.pone.0130834

March, J. (2020). No Effect of Semantic Congruency in a Masked Congruency Paradigm [Master thesis, University of Groningen]. https://fse.studenttheses.ub.rug.nl/23762/

Martens, V. E. G., \& de Jong, P. F. (2006). The effect of word length on lexical decision in dyslexic and normal reading children. Brain and Language, 98(2), 140-149. https://doi.org/10.1016/j.bandl.2006.04.003

Mathôt, S., Grainger, J., \& Strijkers, K. (2017). Pupillary responses to words that convey a sense of brightness or darkness. Psychological Science, 28(8), 1116-1124. https://doi.org/10.1177/0956797617702699

Mathôt, S., Schreij, D., \& Theeuwes, J. (2012). OpenSesame: An open-source, graphical experiment builder for the social sciences. Behavior Research Methods, 44(2), 314-324. https://doi.org/10.3758/s13428-011-0168-7

Mathôt, S., Sundermann, L., \& Rijn, H. van. (2019). The effect of semantic brightness on pupil size: A replication with Dutch words (p. 689265). https://doi.org/10.1101/689265

Munro, R., Bethard, S., Kuperman, V., Lai, V. T., Melnick, R., Potts, C., Schnoebelen, T., \& Tily, H. (2010). Crowdsourcing and language studies: The new generation of linguistic data. NAACL Workshop on Creating Speech and Language Data With Amazon's Mechanical Turk, 122-130.

Peirce, J. W. (2007). PsychoPy: Psychophysics software in Python. Journal of Neuroscience Methods, 162(1-2), 8-13. https://doi.org/10.1016/j.jneumeth.2006.11.017

Petit, J.-P., Midgley, K. J., Holcomb, P. J., \& Grainger, J. (2006). On the time course of letter perception: A masked priming ERP investigation. Psychonomic Bulletin \& Review, 13(4), 674-681. https://doi.org/10.3758/BF03193980 
Pinet, S., Zielinski, C., Mathôt, S., Dufau, S., Alario, F.-X., \& Longcamp, M. (2017). Measuring sequences of keystrokes with jsPsych: Reliability of response times and interkeystroke intervals. Behavior Research Methods, 49(3), 1163-1176. https://doi.org/10.3758/s13428-016-0776-3

Pulvermüller, F. (2013). How neurons make meaning: Brain mechanisms for embodied and abstract-symbolic semantics. Trends in Cognitive Sciences, 17(9), 458-470. https://doi.org/10.1016/j.tics.2013.06.004

R Core Team. (2014). R: A Language and Environment for Statistical Computing. R Foundation for Statistical Computing. http://www.R-project.org/

Reimers, S., \& Stewart, N. (2014). Presentation and response timing accuracy in Adobe Flash and HTML5/JavaScript Web experiments. Behavior Research Methods, 47(2), 309-327. https://doi.org/10.3758/s13428-014-0471-1

Sauter, M., Draschkow, D., \& Mack, W. (2020). Building, hosting and recruiting: A brief introduction to running behavioral experiments online. Brain Sciences, 10(4), 251. https://doi.org/10.3390/brainsci10040251

Seabold, S., \& Perktold, J. (2010). Statsmodels: Econometric and statistical modeling with python. Proceedings of the 9th Python in Science Conference, 57, 61.

Stahl, C. (2006). Software for generating psychological experiments. Experimental Psychology, 53(3), 218-232. https://doi.org/10.1027/1618-3169.53.3.218

Stoet, G. (2010). PsyToolkit: A software package for programming psychological experiments using Linux. Behavior Research Methods, 42(4), 1096-1104. https://doi.org/10.3758/BRM.42.4.1096

Vadillo, M. A., \& Garaizar, P. (2016). The effect of noise-induced variance on parameter 
recovery from reaction times. BMC Bioinformatics, 17, 147.

https://doi.org/10.1186/s12859-016-0993-x

Vogt, A., Hauber, R., Kuhlen, A. K., \& Rahman, R. A. (2021). Internet-based language production research with overt articulation: Proof of concept, challenges, and practical advice. Behavior Research Methods. https://doi.org/10.3758/s13428-021-01686-3

Wagenmakers, E.-J., Wetzels, R., Borsboom, D., Maas, H. L. J. van der, \& Kievit, R. A. (2012). An agenda for purely confirmatory research. Perspectives on Psychological Science, 7(6), 632-638. https://doi.org/10.1177/1745691612463078

Wilschut, T., Sense, F., van der Velde, M., Fountas, Z., Maaß, S. C., \& van Rijn, H. (2021). Benefits of Adaptive Learning Transfer From Typing-Based Learning to Speech-Based Learning. Frontiers in Artificial Intelligence, 4, 780131. https://doi.org/10.3389/frai.2021.780131

Zhou, C., Lorist, M. M., \& Mathôt, S. (2021). Categorical bias in visual working memory: The effect of memory load and retention interval. https://osf.io/puq4v/44 